\title{
Niveles de Razonamiento de Van Hiele en Estudiantes de Séptimo Grado
}

\author{
Van Hiele Levels of Reasoning in Seventh Grade Students
}

DOI: $10.46932 / \operatorname{sfjdv3n1-050}$

Received in: Jan 30st, 2021

Accepted in: Feb 1th, 2022

\section{Yelihsa López Cuadrado}

Ingeniera en Minas. Universidad Pedagógica y Tecnológica de Colombia (Seccional Sogamoso).

Estudiante de maestría en educación. Universidad de Pamplona.

Correo electrónico: yelihsa.lopez@unipamplona.edu.co

\author{
Matilde Bolaño Garcia \\ Ph.D. En educación. Docente e investigadora de la universidad del Magdalena. \\ Correo electrónico: matilde.bolano@unipamplona.edu.co
}

\begin{abstract}
RESUMEN
La geometría es parte primordial de la cotidianidad de los estudiantes, hace parte de su entorno, pero la mayoría de las veces se deja de lado y no se le presta la importancia necesaria que tiene en el desarrollo de las habilidades matemáticas y de otras asignaturas en los estudiantes. Esta investigación se centra en identificar los niveles de razonamiento de Van Hiele predominantes en un grupo de estudiantes del grado séptimo de la Institución Educativa San Juan del Córdoba del municipio de Ciénaga Magdalena. La metodología fue de tipo cualitativa, con un diseño de investigación acción, siguiendo una serie de fases, que permitieron la recolección, la aplicación, el análisis y la interpretación de los datos, con la ayuda de la implementación de instrumentos como una prueba diagnóstica que permitió evidenciar las falencias de los estudiantes en cada uno de los niveles de razonamiento que propone la teoría antes mencionada, permitiendo identificar los diferentes problemas propios del aprendizaje de los estudiantes, con el propósito de construir una serie de secuencias didácticas basadas en estos resultados y con ello desarrollar estrategias innovadoras para la enseñanza.
\end{abstract}

Palabras clave: Geometría, pensamiento espacial, Teoría de Van Hiele

\begin{abstract}
Van Hiele's Levels of Reasoning in Seventh Grade Students

Geometry is an essential part of the daily life of students, it is part of their environment, but most of the time it is left aside and is not given the necessary importance it has in the development of mathematical skills and other subjects in students. This research focuses on identifying the predominant levels of Van Hiele's reasoning in a group of seventh grade students of the San Juan del Córdoba Educational Institution in the municipality of Ciénaga Magdalena. The methodology was qualitative, with an action research design, following a series of phases, which allowed the collection, application, analysis and interpretation of the data, with the help of the implementation of instruments such as a diagnostic test that allowed evidencing the students' deficiencies in each of the levels of reasoning proposed by the aforementioned theory, allowing identifying the different problems of the students' learning, with the purpose of building a series of didactic sequences based on these results and thus developing innovative strategies for teaching.
\end{abstract}

Keywords: Geometry, spatial thinking, Van Hiele's theory. 


\section{INTRODUCCIÓN}

La geometría como el idioma universal de los seres humanos, permite describir y construir su propia realidad, así como también, transmitir la percepción que éste tiene de ella, a otros individuos. Para (Vargas y Gamboa, 2013) es uno de los temas que tiene más importancia en la matemática ya que se relaciona de manera directa o indirecta, con múltiples actividades que se realizan ya sea para el progreso de la sociedad, el estudio o para la recreación.

Conjuntamente la geometría contribuye con el desarrollo de habilidades cognitivas de los individuos como: la visualización, el pensamiento crítico, la intuición, la resolución de problemas, conjeturas, razonamiento deductivo y argumentación lógica en diferentes procesos como pruebas y demostraciones (Jones, 2002). Sumando a esto, la enseñanza de la geometría se concentra, en la memorización de conceptos y su aplicación, sin que el estudiante pueda llegar a una conceptualización más allá de lo que sus propias capacidades se lo permitan. (Barrantes, 2002).

De la misma manera encontramos que la enseñanza de la geometría la hemos limitado sólo al hecho de la conceptualización de figuras y dibujarlas sobre un papel; en la mayoría de los casos, los alumnos no las relacionan con objetos de su cotidianidad, con formas y/u ejemplos reales, que les permita captar de mejor manera los contenidos desarrollados; generalmente las clases de geometría se dictan de manera abstracta, en consecuencia la enseñanza de la geometría debe plantear aquellos contenidos útiles en el futuro desarrollados mediante una metodología dinámica en la que el alumno realice razonamientos, representaciones, relaciones y resolución de actividades. (Barrantes, Balletbo y Fernández, 2014) lo que debe conllevarnos al desarrollo estrategias innovadoras en el proceso de su enseñanza.

Así mismo, un gran porcentaje de actividades desarrolladas en clase suelen ser individualistas, monótonas, aburridas, donde no se promueve el trabajo entre pares limitándose solo a preguntar, y no a cuestionarse por su proceso de aprendizaje, dando conocimientos como verdades absolutas. (Escobar, 2015). Según esto, la enseñanza de la geometría ha estado vinculada con modelos pedagógicos tradicionales, mecanicistas, comunicado a través de distintas generaciones de profesores de matemáticas. Para (Labarrera, 2016) esto ha provocado, que la práctica de la enseñanza de las matemáticas se incline hacia aquellos temas considerados más accesibles, más importantes para la enseñanza, según el criterio del docente, por lo que, algunos temas de geometría se ven relegados, al ser considerados más complejos o poco importantes.

Conjuntamente Fripp (2010), considera la geometría un campo del conocimiento matemático fundamental en la construcción del hombre; no obstante, se hace muy común dejar a la geometría relegada, restándole la importancia que esta se merece, arrojando como consecuencia, resultados negativos en las pruebas externas aplicadas a los estudiantes. Sumado a lo anterior dentro de la institución educativa en 
estudio, la enseñanza de la geometría presenta una baja intensidad horaria semanal, acompañada de una metodología que no es acorde con las exigencias de la actualidad.

Debido a lo expuesto anteriormente se propuso aplicar la Teoría de Van Hiele como estrategia pedagógica en busca ya que dicho modelo busca orientar a los docentes para que sus alumnos mejoren las cualidades del razonamiento matemático, con la premisa de que ellos deben pasar por una serie de niveles, para lo cual según Vargas (2013) deben dominar el nivel en que se encuentran y así pasar al nivel inmediato superior, el estudiante debe cumplir ciertos procesos de logro y aprendizaje. Este modelo distribuye el conocimiento escalonadamente en cinco niveles de razonamiento secuenciales y ordenados.

Es importante señalar el significado que se le da a la palabra enseñar en el Modelo de Van Hiele. Se ha visto cómo dicha propuesta se caracteriza por analizar los procesos del pensamiento geométrico, por lo tanto, su énfasis no está en la asimilación de contenidos y destrezas, sino en el proceso de entendimiento de conceptos y en la manera como perfeccionamos las maneras de razonar matemáticamente. Por lo anterior la palabra enseñar no debemos interpretarla con el sentido que habitualmente se le da en el ámbito educativo: enseñar definiciones y contenidos a partir de sus propiedades.

Queda entonces por señalar que la palabra enseñar debe entenderse como el proceso de desarrollar en los estudiantes maneras innovadoras de razonamiento, las cuales provienen de estructuras mentales novedosas y más complejas que las precedentes, y que sólo pueden ser construidas por el mismo estudiante, a partir de su propia experiencia.

Por lo anterior, se propuso identificar los niveles de razonamiento de Van Hiele predominantes en un grupo de estudiantes del grado séptimo de la Institución Educativa San Juan del Córdoba del municipio de Ciénaga Magdalena, y como éste coadyuda al mejoramiento de los resultados de las pruebas externas, aplicando una prueba diagnóstica que permita conocer los diferentes niveles de razonamiento en que se encuentran los estudiantes.

\subsection{DESARROLLO DEL PENSAMIENTO ESPACIAL}

Dentro de los sistemas geométricos es importante el desarrollo de pensamiento espacial definido como el conjunto de procesos cognitivos mediante los cuales se construyen y se manipulan representaciones mentales de los objetos del espacio, las relaciones entre ellos, sus transformaciones y sus diversas traducciones a representaciones materiales, usado para representar y manipular información en el aprendizaje y en la resolución de problemas. (Alonso, 2011).

Según el Ministerio de Educación Nacional (1998), en su Guía de Estándares Básicos de Competencias, del área de Matemática, definió el pensamiento espacial y los sistemas geométricos como 
ese conjunto de procesos cognitivos mediante los cuales se construyen y se manipulan las representaciones mentales de los objetos en el espacio, las relaciones entre ellos y sus representaciones; dándole importancia a las propiedades de los cuerpos, es necesario tener claro que cuando se abordan estos conceptos se deben tener en cuenta su evolución y la manera como se adquieren, por lo cual este pensamiento es considerado parte esencial del desarrollo de las habilidades matemáticas que serán implementadas por las personas a lo largo de los años, para resolver problemas matemáticos no rutinarios.

Continuando con el concepto de geometría se puede ver como esa área de las matemáticas es la encargada de estudiar idealizaciones del espacio con base en las características y medidas de las formas geométricas, se puede decir que se preocupa por problemas métricos como cálculos de área y diámetro de figuras planas de la superficie y volúmenes de cuerpos sólidos y tienen como propósito describir, clasificar y estudiar cuales son las propiedades de las figuras geométricas.

\title{
1.2 NIVELES DE RAZONAMIENTO SEGÚN VAN HIELE
}

Según el modelo de Van Hiele, los diferentes niveles de razonamiento permiten identificar problemas de aprendizaje y se plantea como una estrategia para que los docentes puedan mejorar la calidad del razonamiento matemático de los estudiantes. Las fases propuestas plantean definir la organización del proceso educativo, lo que permitirá a los estudiantes construir sistemas conceptuales y habilidades que les permitan avanzar en el razonamiento a un nivel superior. En este sentido, Fouz (2006) citado por Vargas (2013) afirma:

\begin{abstract}
Al subir de nivel se hacen explícitos en el estudiante los conocimientos que eran implícitos en el nivel anterior, lo cual indica que va aumentando de esta manera el grado de comprensión y dominio del conocimiento. Esto hace que los objetos de trabajo de este nivel superior sean extensiones de aquellos del nivel anterior. (p. 81-82).
\end{abstract}

De acuerdo con Vargas (2013), categoriza el modelo de Van Hiele en cinco niveles de razonamiento geométrico ordenados en forma numerada de uno a cinco, los cuales se pueden en observar en la tabla no. 1 
Tabla no. 1 Niveles de razonamiento de Van Hiele.

\begin{tabular}{|c|c|}
\hline \multicolumn{2}{|r|}{ NIVELES DE RAZONAMIENTO DE VAN HIELE } \\
\hline NIVELES & DESCRIPCIÓN \\
\hline $\begin{array}{c}\text { Nivel } 1 \\
\text { Reconocimiento }\end{array}$ & $\begin{array}{l}\text { Los estudiantes no definen correctamente las propiedades de las figuras } \\
\text { geométricas, presentan dificultades al ordenarlas, compararlas, describirlas e } \\
\text { incluso identificarlas, en este nivel perciben las figuras de forma general como } \\
\text { una unidad, solo las describen por su apariencia física, las figuras geométricas } \\
\text { son objetos individuales por lo que no pueden generalizar, ni reconocer las } \\
\text { cualidades comunes a dos figuras de una misma clase. A pesar de poder } \\
\text { identificar partes de alguna figura, se les dificulta analizarla de acuerdo a sus } \\
\text { componentes, no tienen claro que las propiedades de ella determinan sus } \\
\text { características y clasificación. Los estudiantes no cuentan con un lenguaje } \\
\text { apropiado. }\end{array}$ \\
\hline $\begin{array}{l}\text { Nivel } 2 \\
\text { Análisis }\end{array}$ & $\begin{array}{l}\text { Los estudiantes reconocen que las formas geométricas se forman de partes y } \\
\text { además poseen cualidades matemáticas, pueden describir sus propiedades, pero } \\
\text { de manera informal, sin relacionarla con las de otras. Pueden comparar figuras } \\
\text { geométricas sólo si se les menciona cual es el empleo de las propiedades de sus } \\
\text { respectivas partes. No comprenden que es una demostración matemática. Los } \\
\text { estudiantes utilizan un lenguaje adecuado para referirse a las partes de las } \\
\text { figuras, por ejemplo en el caso de los ángulos. }\end{array}$ \\
\hline $\begin{array}{c}\text { Nivel } 3 \\
\text { Clasificación }\end{array}$ & $\begin{array}{l}\text { Los estudiantes reconocen que algunas características se deducen de otras, son } \\
\text { capaces de deducir sus implicaciones, esto pueden hacerlo de un solo paso, } \\
\text { aunque no son capaces de comprender como un todo el significado que han } \\
\text { obtenido de su deducción, están en capacidad de comprender los pasos } \\
\text { individuales que se adelantan en el razonamiento lógico formal, pero carecen de } \\
\text { la capacidad de comprender la estructura de una demostración y desconocen } \\
\text { cómo se construyen a partir de las premisas estudiadas. Razonan de acuerdo con } \\
\text { el sistema lógico deductivo, lo que no equivale a razonar con base en la lógica } \\
\text { formal. Realizan razonamientos deductivos no formales, con el uso de las reglas } \\
\text { lógicas, son capaces de entender las demostraciones formales explicadas por el } \\
\text { docente o los textos. Valoran el papel que desempeñan los conceptos, así } \\
\text { mismo, se les facilita dar definiciones verídicas desde el punto de vista } \\
\text { matemático. }\end{array}$ \\
\hline $\begin{array}{c}\text { Nivel } 4 \\
\text { Deducción Formal }\end{array}$ & $\begin{array}{l}\text { Los estudiantes entienden y realizan raciocinios lógicos formales. Pueden } \\
\text { comprender las demostraciones, compuestas por distintas etapas, y pueden } \\
\text { aceptar que son necesarias y les permite la verificación de la veracidad que } \\
\text { pueda tener una afirmación, plantean conjeturas y buscan de verificarlas de } \\
\text { manera deductiva, construyen sus propias demostraciones, no solo las } \\
\text { memorizan, también están en capacidad de plantear demostraciones de diversas } \\
\text { formas. Pueden aceptar que existen definiciones equivalentes de un mismo } \\
\text { concepto, comprende cual es la estructura de axiomas que caracterizan el } \\
\text { pensamiento matemático. Plantean argumentos deductivos formales, pero no } \\
\text { investigan en qué consisten los sistemas axiomáticos. }\end{array}$ \\
\hline $\begin{array}{l}\text { Nivel } 5 \\
\text { Rigor }\end{array}$ & $\begin{array}{l}\text { Los estudiantes alcanzan su más alto nivel de rigor matemático, de acuerdo a } \\
\text { los criterios establecidos actualmente, pueden prescindir de soportes concretos y } \\
\text { así desarrollar sus actividades matemáticas, ya aceptan que existen sistemas } \\
\text { axiomáticos diferentes y hacen usos de ellos, además pueden establecer } \\
\text { comparaciones. }\end{array}$ \\
\hline
\end{tabular}

Fuente: Elaboración propia, con base en Vargas (2013)

\section{METODOLOGÍA}

La investigación se llevó a cabo con un enfoque cualitativo, con un método de investigación acción, como una forma de entender la enseñanza, como un proceso de continua búsqueda y que conlleva a entender el oficio docente, integrando la reflexión y el trabajo intelectual en el análisis de las experiencias que se realizan. (Bauselas, 2004). Esta investigación se aplicó a un grupo de estudiantes de 
séptimo grado de la I. E. San Juan Del Córdoba del municipio de Ciénaga Magdalena, en cuanto al proceso metodológico la investigación contó con una serie de fases de diseño, desarrollo y evaluación, detallados en la siguiente imagen 1.

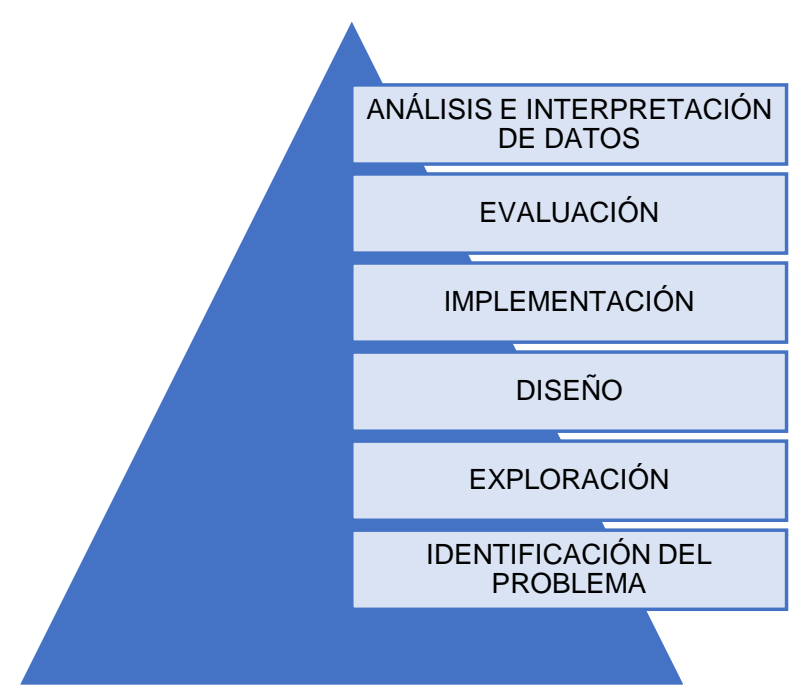

FUENTE: Bolaño M. (2019)

Por otro lado, con el fin de identificar los niveles de razonamiento en que se encuentran los estudiantes al iniciar la investigación se realizó una prueba diagnóstica tomando como base una prueba elaborada por el Dr. Zalman Usiskin (1982) y sus colaboradores que permitió evidenciar las falencias que tenían los estudiantes en cuanto a los conceptos de geometría en los tres primeros niveles de razonamiento de la teoría de Van Hiele, que se pueden evidenciar en la imagen 2
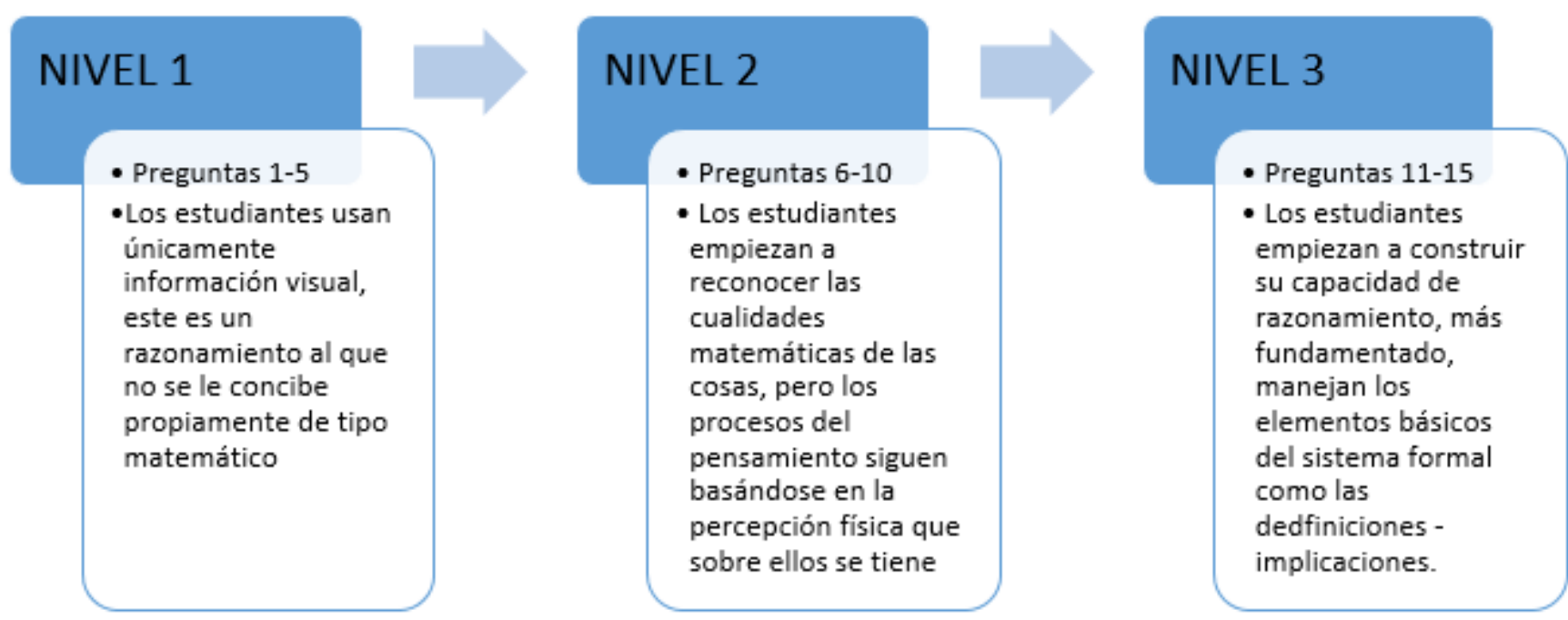

Fuente: Elaboración propia. 
Con base a estos resultados realizar el diseño de la secuencia didáctica a implementar, durante todo el proceso se recogieron las vivencias de los estudiantes en las mediaciones por medio de los diarios de campo, que permitieron identificar situaciones de importancia en el desarrollo de los lineamientos; para finalizar el proceso se realizó la misma prueba inicial que permitió conocer los resultados de la estrategia implementada.

\section{RESULTADOS}

Para la planeación de las actividades y diseño de los instrumentos se tuvieron en cuenta los planteamientos del modelo de Van Hiele, en un cuestionario semiestructurado, con preguntas de selección múltiple el cual se puede observar detalladamente en el anexo no. 1, en el cual se busca que el estudiante recupere información implícita en el contenido del problema y resuelva de acuerdo con sus conocimientos matemáticos iniciales, este proceso se llevó a cabo un proceso de manera ordenada conformado por 15 preguntas que permitieron evaluar los primeros tres niveles de razonamiento conocidos como nivel 1 o de reconocimiento donde los estudiantes perciben las figuras de manera general como una unidad, aquí solo se usa únicamente información visual; el nivel 2 o de análisis en donde los estudiantes empiezan a reconocer las cualidades matemáticas de las cosas, pero los procesos siguen basándose en la percepción física que sobre ellos se tiene. El nivel 3 o de clasificación, ya empiezan a construir su capacidad de razonamiento más fundamentado y a manejar los elementos más básicos del sistema formal como las definiciones - implicaciones.

La prueba diagnóstica, se realizó con la ayuda del programa Socrative, que evaluó los tres primeros niveles de razonamiento de Van Hiele, que consto de 15 preguntas relacionadas de la siguiente manera:

- Nivel 1. Reconocimiento: En las cinco primeras preguntas se pudo observar que los estudiantes presentaban dificultad a la hora de identificar las figuras geométricas que se les presenta, algunas veces con el solo hecho de girar una figura, empezaban a tener dudas de la forma general de dicha figura.

- Nivel 2. Análisis: En las preguntas de la 6 a la 10 se observó mayor grado de dificultad, la mayoría de respuestas fueron incorrectas, los estudiantes tuvieron problemas a la hora de analizar las cualidades y las partes de las figuras que se les presentaron.

- Nivel 3. Clasificación: De las preguntas 11 a la 15 los estudiantes no pudieron realizar conjeturas sobre los enunciados presentados, lo que demuestra las dificultades en el momento de realizar razonamientos lógicos en cuanto a las figuras mostradas en la prueba. 
La prueba se realizó de manera sincrónica con seis estudiantes, proceso en el cual se les hizo acompañamiento en todo momento, los otros dos estudiantes presentaron problemas de conectividad por lo cual se les dieron instrucciones y la pudieron realizar en diferente horario. Una vez finalizado este proceso se procedió a procesar los datos arrojados por el programa Socrative en un cuadro de Excel, que se puede ver en la Tabla no. 2 que se presenta a continuación. 
Tabla no. 2. Resultados de la Prueba Diagnóstica

\begin{tabular}{|c|c|c|c|c|c|c|c|c|c|c|c|c|c|c|c|c|c|}
\hline $\begin{array}{l}\text { Nombre del } \\
\text { estudiante }\end{array}$ & (\%) & $\begin{array}{l}\text { Puntua- } \\
\text { ción (\#) }\end{array}$ & $\begin{array}{l}\text { Punto } \\
1\end{array}$ & $\begin{array}{l}\text { Punto } \\
2\end{array}$ & $\begin{array}{l}\text { Punto } \\
3\end{array}$ & $\begin{array}{l}\text { Punto } \\
4\end{array}$ & $\begin{array}{l}\text { Punto } \\
5\end{array}$ & $\begin{array}{l}\text { Punto } \\
6\end{array}$ & $\begin{array}{l}\text { Punto } \\
7\end{array}$ & $\begin{array}{l}\text { Punto } \\
8\end{array}$ & $\begin{array}{l}\text { Punto } \\
9\end{array}$ & $\begin{array}{l}\text { Punto } \\
10\end{array}$ & $\begin{array}{l}\text { Punto } \\
11\end{array}$ & $\begin{array}{l}\text { Punto } \\
12\end{array}$ & $\begin{array}{l}\text { Punto } \\
13\end{array}$ & $\begin{array}{l}\text { Punto } \\
14\end{array}$ & $\begin{array}{l}\text { Punto } \\
15\end{array}$ \\
\hline & & 15 & $\begin{array}{l}1 \\
\text { Punto }\end{array}$ & $\begin{array}{l}1 \\
\text { punto }\end{array}$ & $\begin{array}{l}1 \\
\text { punto }\end{array}$ & $\begin{array}{l}1 \\
\text { punto }\end{array}$ & $\begin{array}{l}1 \\
\text { punto }\end{array}$ & $\begin{array}{l}1 \\
\text { punto }\end{array}$ & $\begin{array}{l}1 \\
\text { punto }\end{array}$ & $\begin{array}{l}1 \\
\text { punto }\end{array}$ & $\begin{array}{l}1 \\
\text { punto }\end{array}$ & $\begin{array}{l}1 \\
\text { punto }\end{array}$ & $\begin{array}{l}1 \\
\text { punto }\end{array}$ & $\begin{array}{l}1 \\
\text { punto }\end{array}$ & $\begin{array}{l}1 \\
\text { punto }\end{array}$ & $\begin{array}{l}1 \\
\text { punto }\end{array}$ & $\begin{array}{l}1 \\
\text { punto }\end{array}$ \\
\hline Informante 1 & 20 & 3 & B. & E. & A. & B. & C. & A. & D. & C. & C. & E. & E. & A. & E. & E. & A. \\
\hline Informante 2 & 26,67 & 4 & B. & E. & A. & B. & C. & E. & A. & A. & B. & B. & C. & A. & B. & B. & C. \\
\hline Informante 3 & 20 & 3 & B. & A. & A. & A. & D. & c. & c. & B. & A. & c. & A. & C. & B. & A. & B. \\
\hline Informante 4 & 26,67 & 4 & B. & c. & A. & B. & E. & C. & A. & B. & A. & B. & C. & C. & E. & D. & D. \\
\hline Informante 5 & 13,33 & 2 & C. & A. & A. & A. & A. & C. & A. & B. & A. & C. & C. & B. & E. & E. & A. \\
\hline Informante 6 & 26,67 & 4 & B. & E. & c. & B. & C. & D. & A. & C. & B. & D. & A. & A. & E. & c. & D. \\
\hline Informante 7 & 40 & 6 & B. & D. & C. & B. & C. & B. & C. & A. & A. & B. & D. & A. & E. & E. & A. \\
\hline Informante 8 & 46,67 & 7 & B. & D. & C. & B. & C. & B. & C. & A. & A. & B. & C. & A. & E. & E. & A. \\
\hline $\begin{array}{l}\text { Puntuación de } \\
\text { clase }\end{array}$ & 27,5 & 4,13 & 87,5 & 25 & 37,5 & 75 & 12,5 & 25 & 0 & 37,5 & 12,5 & 12,5 & 50 & 12,5 & 0 & 12,5 & 12,5 \\
\hline
\end{tabular}

Fuente: Elaboración propia a partir de implementació n del programa Socrative. 
A continuación, en la tabla no. 3 se muestra un resumen de las respuestas correctas obtenidas en cada nivel, durante la prueba diagnóstica

Tabla no. 3 Respuestas correctas obtenidas en la prueba diagnóstica

\begin{tabular}{|c|c|c|c|}
\hline \multicolumn{5}{|c|}{ NÚMERO DE RESPUESTAS EN CADA NIVEL } \\
\hline INFORMANTE & NIVEL 1 & NIVEL 2 & NIVEL 3 \\
\hline 1 & 2 & 1 & 0 \\
\hline 2 & 2 & 1 & 1 \\
\hline 3 & 1 & 0 & 2 \\
\hline 4 & 3 & 0 & 1 \\
\hline 5 & 0 & 0 & 2 \\
\hline 6 & 3 & 1 & 0 \\
\hline 7 & 4 & 2 & 7 \\
\hline $\begin{array}{l}\text { Total respuestas } \\
\text { correctas } \\
\text { nivel cada }\end{array}$ & 4 & 2 & \\
\hline
\end{tabular}

Analizando los resultados de las Tablas no. 2 y no. 3, las primeras 5 preguntas corresponden al primer nivel de reconocimiento, donde respondieron 19 respuestas correctas, aquí se pudo observar que los estudiantes presentan falencias a la hora de identificar las figuras geométricas que se les presentan, con el sólo hecho de girarlas, empiezan a tener dudas con respecto a la forma de estas.

A partir de la pregunta 6 a la 10, respondieron 7 respuestas correctas, se buscó trabajar el nivel de análisis, donde se observó, mayor grado de dificultad, la mayoría de respuestas fueron erróneas mostrando las falencias que tienen los estudiantes a la hora de realizar análisis sobre las cualidades, las partes, mediante la observación de las figuras.

Para finalizar con la prueba de las preguntas 11 a la 15, respondieron 7 respuestas correctas, se pudo observar que los estudiantes tuvieron grandes inconvenientes a la hora de realizar conjeturas sobre enunciados, lo que demuestra las dificultades a la hora de realizar razonamientos lógicos en cuanto a las figuras mostradas en la prueba

Estos resultados evidencian las grandes dificultades que tienen los estudiantes, en estos 3 niveles evaluados lo que muestra la gran dificultad que tienen ellos en el desarrollo del pensamiento espacial, se debe tener en cuenta que gran parte de esas falencias se deben a una falta de saberes previos que deberían tener sobre los temas evaluados, si se analiza que el año inmediatamente anterior fue un año fuera de lo común que no permitió cumplir con la intensidad y la programación dispuesta dentro de los planes de área de la institución debido a la pandemia del covid-19.

Sumado a todo esto, los estudiantes tienen unos contextos sociales, económicos y culturales que no favorecieron el buen desarrollo de las clases, con todo esto analizado se puede clasificar a los 
estudiantes dentro de un nivel de razonamiento 1 o de reconocimiento, caracterizado por ser visuales en sus apreciaciones, son muy generales, tienen conceptos muy básicos, lo que sirve de base para elaborar unas secuencias didácticas enfocadas en ejes temáticos básicos del grado séptimo para fortalecer y lograr de una manera progresiva todas las habilidades geométricas y así poder alcanzar el nivel deseado.

\section{CONCLUSIONES}

Partiendo del objetivo de identificar los diferentes niveles de razonamiento de Van Hiele, se realizó la prueba diagnóstica elaborada por el matemático Usiskin que mostró las dificultades de los estudiantes al dar solución a las preguntas realizadas, evidenciando que no son capaces de reconocer figuras geométricas planas, así como realizar análisis de sus propiedades y sacar conjeturas al respecto, clasificándolos en un nivel 1 de razonamiento muy visual y con conocimientos muy básicos.

\section{DECLARACIÓN DE NO CONFLICTO DE INTERESES}

Ni el(los) autor(es) del artículo presentado ni su(s) familia (res) inmediata(os) tiene(tenemos) un acuerdo financiero o afiliación importante con productos y servicios empleados o abordados en el artículo o cualquier otro posible sesgo potencial en contra de otro producto o servicio. La información es propia, y los datos fueron recabados por los autores. 


\section{REFERENCIAS}

ALONSO, A. (octubre de 2011). Desarrollo del pensamiento espacial y sistema geométrico e el aprendizaje de los sólidos regulares mediante el modelo de Van Hiele, con los estudiantes de $6^{\circ}$ grado del colegio San José de la comunidad Marista. 12 Encuentro Colombiano de Matemática Educativa llevado a cabo en Quindío, Colombia.

Barrantes, M. (2002). Recuerdos, expectativas y concepciones de los estudiantes para maestro sobre la geometría escolar y su enseñanza-aprendizaje (Tesis de Doctorado). Departamento de Didáctica de la Ciencias Experimentales y de las Matemáticas. Facultad de Educación. Universidad de Extremadura. España.

Barrantes, M., Balletbo, I. y Fernández, M. (noviembre de 2014). Enseñar geometría en secundaria. Congreso Iberoamericano de ciencia, tecnología, innovación y educación.

Bausela Herreras, E. (2004). La docencia a través de la investigación-acción. Revista Iberoamericana De Educación, 35(1), 1-9. https://doi.org/10.35362/rie3512871

ESCOBAR, B. (2015). El método socrático y el modelo de Van Hiele. Lecturas Matemáticas, 24, pp. 99-121. Universidad de Antioquia. Medellín. Recuperado de http://www.scm.org.co/aplicaciones/revista/Articulos/733.pdf

Fouz, F. (2006). Test geométrico aplicando el Modelo de Van Hiele. Sigma Revista de Matemáticas 28(5), 33-58. Retrieved from http://www.hezkuntza.ejgv.euskadi.net/sigma_28/5_test_geometrico

Fripp, A. (2012). Enseñanza de la geometría en la escuela primaria. cuadernos de investigación educativa, 2(18), 5564. Recuperado de: https://www.ort.edu.uy/ie/cuadernos

JONES, K. (2002). Problemas en la enseñanza y el aprendizaje de la geometría. En L. Haggarty (Ed.), Aspectos de Enseñanza de las Matemáticas Secundarias. Perspectivas sobre la práctica. Ed. Routledge Falmer, Londres.

LABARRERA, (2016). El modelo de desarrollo Van Hiele del pensamiento geométrico, N.T.C.M.: Aprendiendo y enseñando geometría, K12, N.T.C.M., Reston.

Ministerio De Educación Nacional, MEN. (1998). Lineamientos curriculares, Matemáticas. Ed. Cooperativa Magisterio, Bogotá.

Usiskin, Z. (1982). Van Hiele levels and achievement in secondary school geometry. Columbus, EE.UU: ERIC.

VARGAS, G., GAMBOA, R. (2013) El Modelo de Van Hiele y la enseñanza de la geometría. Costa Rica. Revistas Uniciencia. Tomo.1. 


\section{ANEXOS}

Anexo 1. Prueba Diagnóstica

Nombre del estudiante Grado

Esta prueba comprende 15 preguntas. Es normal que no sepa todo lo que se le pregunta.

$\mathrm{Al}$ dar inicio a la prueba realice los siguiente:

a. Leer cada pregunta de manera atenta.

b. Seleccione la respuesta correcta en cada pregunta. Sólo hay una respuesta correcta.

c. Marque con una equis la respuesta correcta.

d. Dispone de 45 minutos para resolver las preguntas.

1. ¿Cuál de las siguientes figuras es un cuadrado?

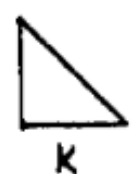

a. K solamente

b. L solamente

c. M solamente

d. L y M solamente

e. Todos son cuadrados

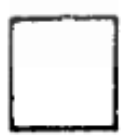

L

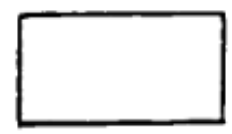

M

2. ¿Cuáles de las siguientes figuras son triángulos?
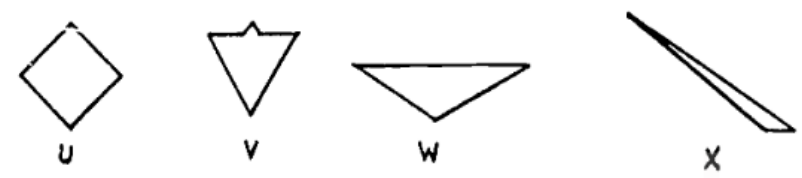

a. Ninguna es un triángulo.

b. V únicamente.

c. W únicamente.

d. W y X únicamente

e. V y W únicamente 
3. ¿Cuáles de las siguientes figuras son rectángulos?
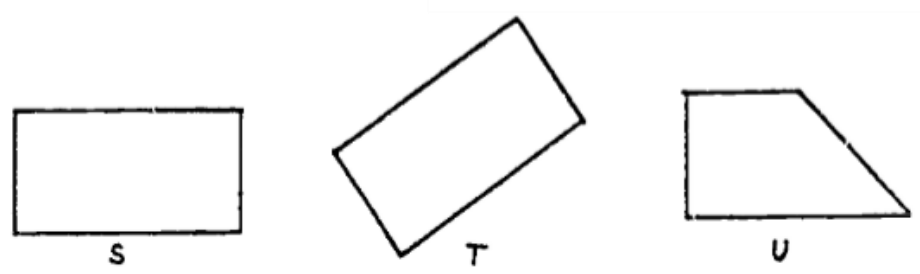
a. $\mathrm{S}$ únicamente.
b. $\mathrm{T}$ únicamente.
c. S y T únicamente
d. S y U únicamente
e. Todas son rectángulos

4. ¿Cuáles de las siguientes figuras son cuadrados?
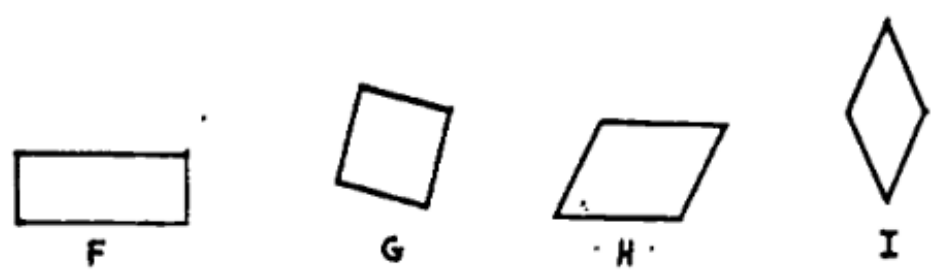
a. Ninguna es un cuadrado
b. $\mathrm{G}$ únicamente.
c. F y $\mathrm{G}$ únicamente
d. G e I únicamente
e. Todas son cuadrados

5. ¿Cuál de las siguientes figuras son paralelogramos?
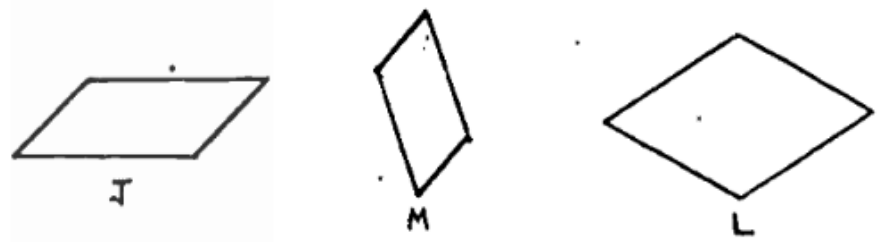
a. J únicamente.
b. L únicamente.
c. J y M únicamente
d. Ninguna es un paralelogramo
e. Todas son paralelogramos 
6. PQRS es un cuadrado

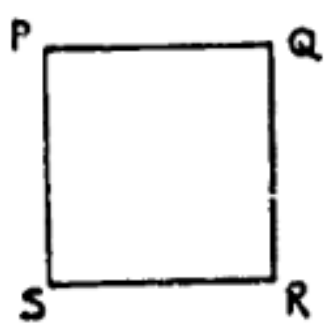

¿Qué relación es verdadera en todos los cuadrados?
a. PR y RS tienen la misma longitud.
b. QS y PR son perpendiculares.
c. PS y QR son perpendiculares.
d. PS y QS tienen la misma longitud.
e. El ángulo Q es mayor que el ángulo $\mathrm{R}$

7. En un rectángulo GHJK, GJ y HK son las diagonales.

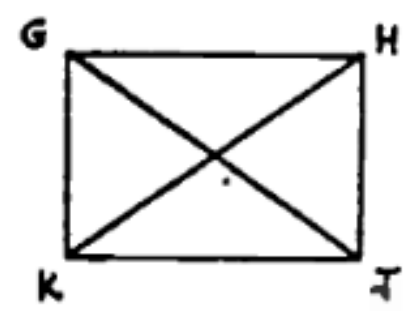

De la afirmación a. hasta la d., ¿Cuál no es cierta en todos los rectángulos?

a. Tienen cuatro ángulos rectos

b. Tienen cuatro lados

c. Las diagonales tienen la misma longitud

d. Los lados opuestos tienen la misma longitud

e. Todas las afirmaciones anteriores son verdades en todos los rectángulos. 
8. Un rombo es una figura de 4 lados con todos los lados de la misma longitud. Aquí hay tres ejemplos:
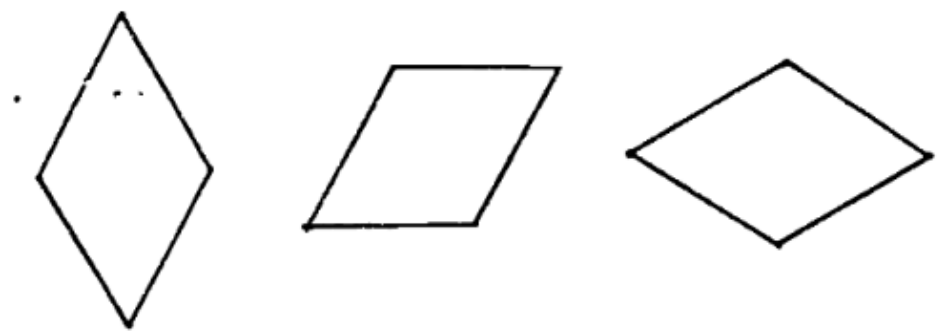

De la afirmación a. hasta la d., ¿cuál no es cierta en todos los rombos?
a. Las dos diagonales tienen la misma longitud.
b. Cada diagonal biseca dos ángulos del rombo.
c. Las dos diagonales son perpendiculares.
d. Los ángulos opuestos tienen la misma medida.
e. Todas las afirmaciones anteriores son verdaderos en todos los rombos.

9. Un triángulo isósceles es un triángulo con dos lados de igual longitud. Aquí hay tres ejemplos,
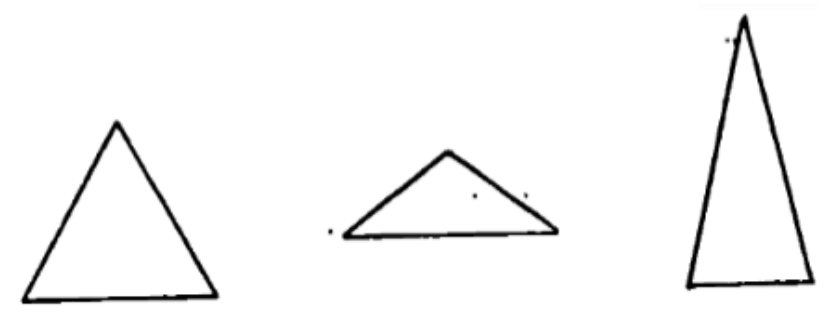

De la afirmación a. hasta la d., ¿cuál no es cierta en todos los triángulos isósceles?

a. Los tres lados deben tener la misma longitud.

b. Un lado debe tener el doble de largo que el otro.

c. Debe haber al menos dos ángulos con la misma medida.

d. Los tres ángulos deben tener la misma medida.

e. Todas las afirmaciones anteriores son verdaderas en todos los triángulos isósceles. 
10. Dos círculos con centros $\mathrm{P}$ y $\mathrm{Q}$ se intersecan en $\mathrm{R}$ y $\mathrm{S}$ para formar una figura PRQS de 4 lados. Aquí se muestran dos ejemplos:
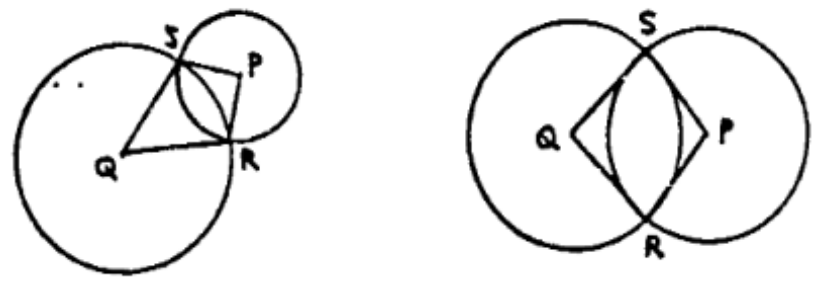

De la afirmación a. hasta la d., ¿cuál nunca es cierta?

a. PRQS tendrá dos pares de lados de igual longitud.

b. PRQS tendrá al menos dos ángulos de igual medida.

c. Las líneas PQ y RS serán perpendiculares.

d. Los ángulos P y Q tendrán la misma medida.

e. Todas las afirmaciones anteriores son verdaderas.

11. Se tienen dos afirmaciones:

Afirmación 1: La figura $\mathrm{F}$ es un rectángulo.

Afirmación 2: La figura $\mathrm{F}$ es un triángulo.

¿Cuál es correcta?

a. Si 1 es verdadero, entonces 2 es verdadero.

b. Si 1 es falso, entonces 2 es verdadero.

c. 1 y 2 no pueden ser ambos verdaderos.

d. 1 y 2 no pueden ser ambos falsos.

e. Ninguna de las anteriores opciones es correcta.

12. Se tienen dos afirmaciones:

Afirmación 1: El triángulo ABC tiene tres lados de la misma longitud.

Afirmación 2: En el triángulo $\mathrm{ABC}$, los ángulos $\mathrm{B}$ y C tienen la misma medida.

¿Cuál es correcta?

a. Las Afirmaciones S y T no pueden ser ambas verdaderas.

b. Si S es verdadero, entonces $\mathrm{T}$ es verdadero,

c. Si $\mathrm{T}$ es verdadero, entonces $\mathrm{S}$ es verdadero. 
d. Si S es falso, entonces T es falso.

e. Ninguna de las anteriores opciones es correcta.

13. ¿Cuál de las siguientes figuras se pueden llamar rectángulos?

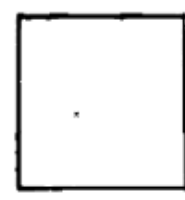

$p$

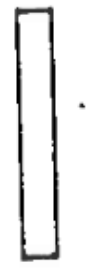

Q.

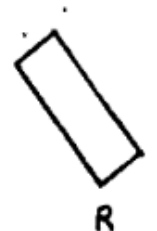

R
a. Todas
b. Q solamente
c. sólo R
d. P y Q solamente
e. Q y R solamente

14. ¿Cuál de las siguientes afirmaciones es verdadera?

a. Todas las propiedades de los rectángulos son propiedades de todos los cuadrados.

b. Todas las propiedades de los cuadrados son propiedades de todos los rectángulos.

c. Todas las propiedades de los rectángulos son propiedades de todos los paralelogramos.

d. Todas las propiedades de los cuadrados son propiedades de todos los paralelogramos.

e. Ninguna de las anteriores afirmaciones es verdadera.

15. ¿Qué tienen todos los rectángulos que no tengan algunos paralelogramos?
a. Lados opuestos iguales
b. Diagonales iguales
c. Lados opuestos paralelos
d. Ángulos opuestos iguales
e. Ninguna de las anteriores afirmaciones es verdadera. 